\title{
Power Factor Analysis of the Linear Motor in Mines
}

\author{
Xianyi Qian ${ }^{\mathrm{a}, *}$ \\ ${ }^{a}$ School of Electronic Information \& Electric Engineering, Changzhou Institute of Technology, CZU, \\ Changzhou, China
}

\begin{abstract}
This paper introduces the structure of linear motor in mines. Analyze the power relation of power-AC -linear motor - vibrant machine, based on this, count the power factor; and make mechanical analysis to the vibrancy, get the power factor, which should be: in the precondition of without collision for the top and bottom magnet, do best to decrease the $\delta_{0}$ to close to $\Delta X_{m}$ ( $\Delta X_{m}$ depends on the technique of the vibrant load), make $K_{\delta}$ close to 1 and $\lambda_{e}$ close to critical maximum $\lambda_{e m}$. It is significantly useful to design linear motor.
\end{abstract}

Index Terms: Linear Motor; Power Factor; Vibration Analysis; Electromagnetic Force; Duty Cycle Analysis

(C) 2011 Published by MECS Publisher. Selection and/or peer review under responsibility of the Research Association of Modern Education and Computer Science

\section{Introduction}

When the computer controlling vibration force fundamental frequency equal to the natural frequency that generate electrical and mechanical resonance, the smaller the excitation force may have a greater amplitude. Therefore, linear motor and its AC device can be used for coal mine and replaced large amplitude rotating machinery vibration motor and mechanical transmission device (eccentric wheel and gear, etc.), to cancel friction bearings and mechanical contact, greatly reducing the mechanical and electrical consumption, extend equipment life.

\section{Power Relations}

As the motor air gap is not only the magnetic field space for mechanical and electrical energy conversion, but also the working air gap for vibration machine reciprocating linear motion, so it is a larger gap width; and since the ratio magnetic energy into mechanical energy is relatively low in a cycle of alternating air-gap width, so power factor is low, coupled with the drive itself, as in the power transfer ratio is less than 1, which makes the grid side of the total power factor is lower than the power factor.

\footnotetext{
* Corresponding author:

| E-mail address: hbxfqxyqxy_123@163.com
} 


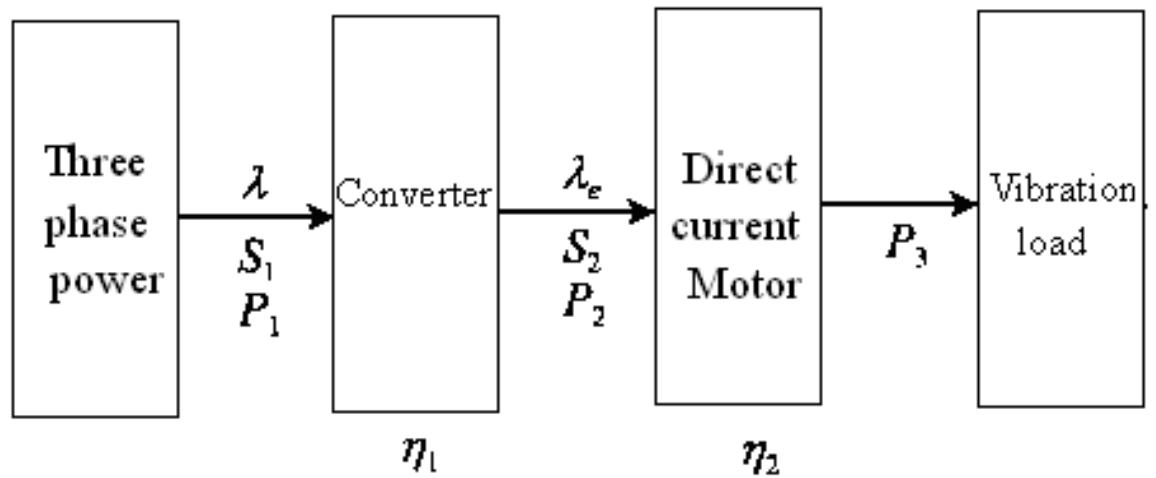

Fig. 1. Power relationship of power - AC devices - linear motors - mechanical vibration

Analysis of linear motor power factor, apparent power converter transmission ratio and overall system power factor, to find the quantitative impact factors of power factor which provide the basis to improve the power factor. From Fig.1 shows the relationship of the every value:

$$
\lambda=\frac{P_{1}}{S_{1}}=\frac{S_{2}}{S_{1}} \cdot \frac{P_{2}}{\eta_{1} \cdot S_{2}}=\frac{1}{\eta_{1}} K_{S} \cdot \lambda_{e}
$$

where $\lambda$ is the total power on the side of the grid; $S_{1} 、 P_{1}$ are the apparent power and the actual reactive power on grid side; $S_{2} 、 P_{2} 、 Q_{2}$ are the motor input apparent power and actual reactive power and virtual power.

\section{Power Factor}

$$
\eta_{1}=\frac{P_{2}}{P_{1}} \text { is AC converter efficiency. } \quad K_{S}=\frac{S_{2}}{S_{1}} \text { is converter apparent power transfer ratio; } \quad \lambda_{e}=\frac{P_{2}}{S_{2}} \text { is }
$$

motor power factor. Because $P_{3}$ is vibration load power, $\mu_{2}$ is motor efficiency, so

$$
\lambda_{e}=\frac{P_{3}}{\eta_{2} \cdot S_{2}}
$$

So (1) can be as below

$$
\lambda=\frac{1}{\eta_{1} \eta_{2}} K_{S} \cdot \frac{P_{3}}{S_{2}}
$$

If need to count $k_{s}, \lambda_{e}$ and $\lambda$, have to count $P_{3}$ and $S_{2}$ 


\section{Vibration Analyses}

By the mechanical analysis shows that the two-mass elastic system for the exciting force in Fig.2 is

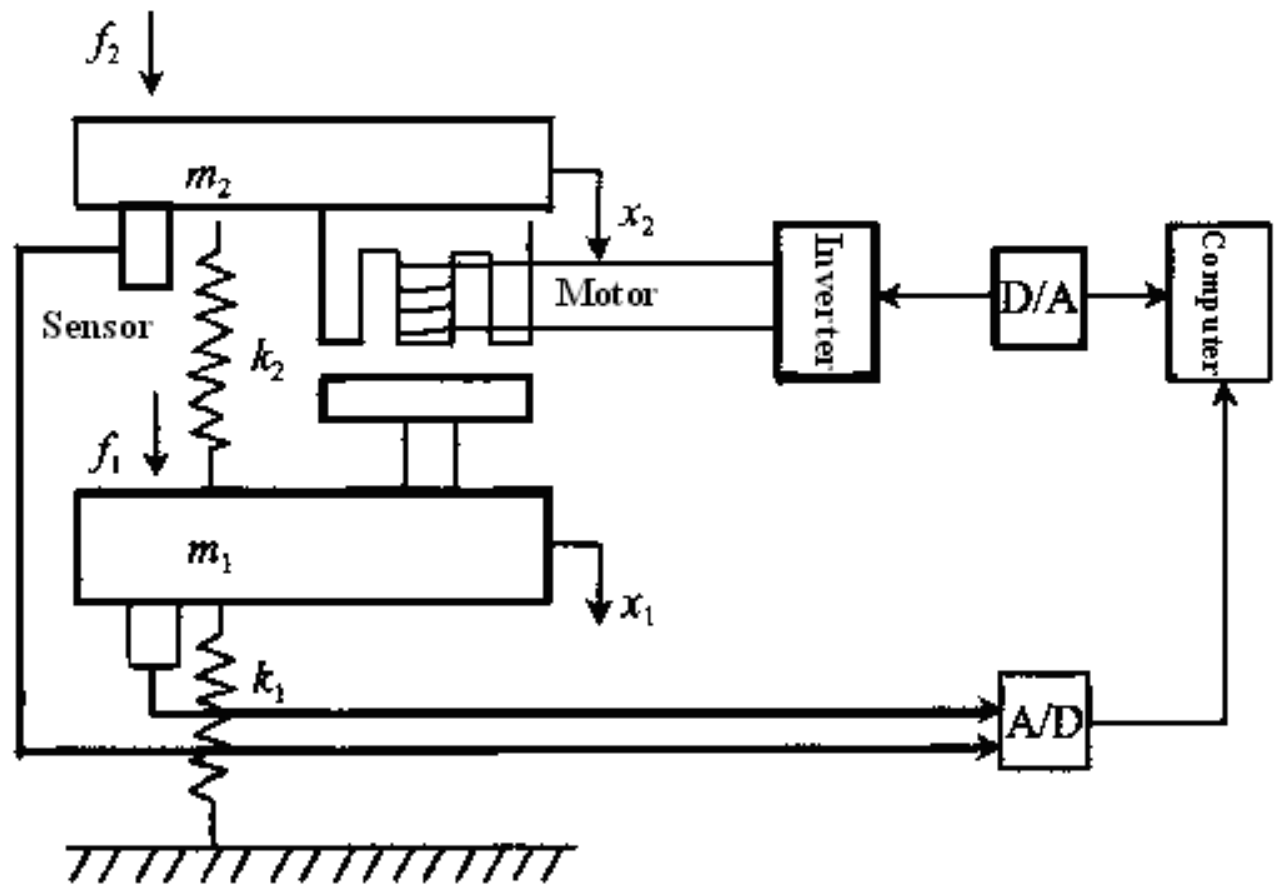

Fig. 2. Computer controlled linear motor drag the double mass sieve system diagram

$f_{2} 、 f_{1}$ are imposed on the upper and lower electromagnetic exciting force screen; $k_{2} 、 k_{1}$ are spring stiffness

$m_{2} 、 m_{1}$ are mass of upper and lower sieve; $x_{2} 、 x_{1}$ are displacement of upper and lower sieve

$$
\left[\begin{array}{l}
f_{1} \\
f_{2}
\end{array}\right]=\left[\begin{array}{c}
F_{m} \cos \omega t \\
-F_{m} \sin \omega t
\end{array}\right]
$$

Double mass vibration displacement is approximately

$$
\left[\begin{array}{l}
x_{1} \\
x_{2}
\end{array}\right]=\left[\begin{array}{c}
x_{1 m} \cos \omega t \\
-x_{2 m} \sin \omega t
\end{array}\right]
$$

Motor steady-state gap width of approximately 


$$
\delta(t)=\delta_{0}+\Delta X_{m} \sin \omega t
$$

All kinds expressions of the above, $F_{m}$ is the amplitude of the electromagnetic exciting force. $\omega$ is for the electrical and mechanical resonance angle frequency; $x_{1 m}, x_{2 m}$ are for the double mass displacement amplitude, $\delta_{0}$ the dynamic level for the position of the gap width of the density, $\Delta x_{m}=x_{1 m}+x_{2 m}$ is for the double mass amplitude sum ( reduce the dynamic stress passed to the foundation, the system resonance to second-order natural frequency).

\section{Analysis of the Electromagnetic Force Duty Cycle}

From faraday's law of electromagnetic induction approximately expression can be obtained

$$
\phi=\frac{1}{N} \int_{0}^{t} u d t=\frac{U m}{N} \int_{0}^{t} u^{0}(t) d t
$$

Where $\mathrm{N}$ is the number of turns the motor windings,

$$
U=U_{m} u^{0}(t)
$$

$U$ for the converter voltage applied to the motor windings, $U_{m}$ is the voltage maximum. $u^{0}(t)$ is

$$
u^{0}(t)= \begin{cases}+1 & 0 \leq t \leq t_{f} \\ -1 & t_{f}<t \leq T\end{cases}
$$

Where $t_{f}$ is the turning time for the voltage from positive to negative, $T=\frac{2 \pi}{\omega}$ for the electrical and mechanical resonance cycle.

As the role of single-chip microprocessor control, only make the electromagnetic force fundamental frequency component and mechanical natural frequency equal and obtain to electrical and mechanical resonance, the second and above harmonic resonance electromagnetic force are small amplitude which can be neglected effect. F for the electromagnetic suction Fourier expansion, the expression for the fundamental electromagnetic force:

$$
F_{1}=-F_{m} \cos \omega t
$$

\footnotetext{
$F_{m}$ is the maximum value of $F_{1}$, get the average power of vibrating machinery
} 


$$
F_{3}=\frac{1}{2} \omega F_{m} \Delta X_{m}
$$

If you only consider the motor air gap reluctance while ignoring iron reluctance. By (6) can get reluctance

$$
R_{\delta}=\frac{2 \delta}{\mu_{0} A}=\frac{2}{\mu_{0} A}\left[\delta_{0}+\Delta X_{m} \sin \omega t\right]
$$

Above formula, A is for the E-type magnet core cross-sectional area. By magnetic Ohm law, consider (7) and (12), we have the current $i$ expression

$$
i=\frac{\phi R_{\delta}}{N}=\frac{2 U_{m}}{N^{2} \mu_{0} A}\left[\delta_{0}+\Delta X_{m} \sin \omega t\right] \int_{0}^{t} u^{0}(t) d t
$$

Current valid value is

$$
I=\sqrt{\frac{1}{T} \int_{0}^{T} i^{2} d t}=\frac{\omega F_{m} K_{t}}{2 U_{m}}
$$

Where

$$
K_{i}=\sqrt{\frac{\pi^{2}}{3}} \delta_{0}^{2}+\sqrt{\left(\frac{\pi^{2}}{6}-\frac{1}{4}\right) \Delta X_{m}^{2}}
$$

Take (14) into the motor apparent power expression $\mathrm{S}_{2}=\mathrm{U}_{\mathrm{m}} \mathrm{I}$, obtain

$$
S_{2}=\frac{1}{2} \omega F_{m} K_{t}
$$

Take (11), (15) and (16) into (2), obtain

$$
\lambda_{e}=\frac{\Delta X_{m}}{\eta_{2} K_{t}}=\frac{\Delta X_{m}}{\sqrt{\frac{\pi^{2}}{3} \delta_{0}^{2}+\left(\frac{\pi^{2}}{6}-\frac{1}{4}\right) \Delta X_{m}^{2}}}=\frac{K_{\delta}}{\eta_{2} \sqrt{\frac{\pi^{2}}{3}+\left(\frac{\pi^{2}}{6}-\frac{1}{4}\right) K_{\delta}^{2}}}
$$

Above 


$$
K_{\delta}=\frac{\Delta X_{m}}{\delta_{0}}
$$

$K_{\delta}$ is the amplitude of the motor duty cycle. Fig. 2 shows that upper and lower magnet of the motor critical collision will occur when $\Delta X_{m}=\delta_{0}$, so must be $\Delta X_{m}<\delta_{0}$, that is $K_{\delta}<1$ to avoid collision. $K_{\delta}=1$ will be substituted into (17) can be obtained the critical maximum of $\lambda_{e}$

$$
\lambda_{e m}=0.462 / \eta_{2}
$$

Because quick vibration of the large amplitude and random variation of the load (by sieving and conveying the material), in the experiment, $K_{\delta}$ must be small to control the magnet will not crash. The motor magnet will no longer crash when $\delta_{0}=12 \mathrm{~mm}$ through the pre-regulator, so when the motor running normally

$$
K_{\delta}=\frac{\Delta X_{m}}{\delta_{0}}=0.533
$$

\section{Conclusions}

$\lambda_{e}=0.6 \lambda_{e m}$ by (17) and (19). In order to improve the power factor, must be guaranteed that the motor upper and lower magnet will not collide to make $\delta_{0}$ as close as possible to the $\Delta X_{m}\left(\Delta X_{m}\right.$ is dependent on the techniques of vibration load), and make $K_{\delta}$ close to 1 , which can make $\lambda_{e}$ close to the critical $\operatorname{maximum}^{\lambda}{ }_{\text {em }}$.

\section{Acknowledgements}

We are very grateful to the Jiangsu Province College Natural Science Foundation of China for the support.

\section{References}

[1] Zhai Xiaofei; Zhang Junhong; Zhao Jinghong. The MATLAB Simulation of the Permanent Magnetic Linear Synchronous Motor and Positioning Experiment[J]. Marine Electric \& Electronic Technology,2006(4)

[2] Chen Yu, Lu Qinfen, Ye Yunyue. Optimal Design of a Long Stator Synchronous Linear Motor[J]. Transactions of China Electrotechnical Society,2003(2)

[3] Shi Jun, Fang Youtong, Ye Yunyue, Jiao Hongxin. Vector Control System Based on Single Neuron PI for Linear Induction Motor[J]. Transactions of China Electrotechnical Society,2003(4) 
[4] Wang Guirong, Wei Wei, Liu Jing. Calculation of Air Flux Density for TR08. Transactions of China Electrotechnical Society,2006(7)

[5] Qiao Mingzhon, Wei Jianghua, Ye Hongchun. Calculation of Exterior Rotor BLDCM Considering Skewed Stator Slot and Rotor Movement[J]. Large Electric Machine and Hydraulic Turbine,2006(2)

[6] Gong Jinguo, Ren Haipeng, Liu Ding. Speed control of brushless DC motor drivers by load torque estimation[J]. Electric Machines and Control,2005(6)

[7] Liu Yongjun, Yang Zhongping. Suspension Technique for Linear Induction Motor[J]. Urban Rapid Rail Transit, 2006(1)

[8] Chen Yangsheng, Lin Youyang. Calculation of Airgap Flux Density in Permanent Magnet Machines[J]. Proceedings of the CSEE, 1994(5) 